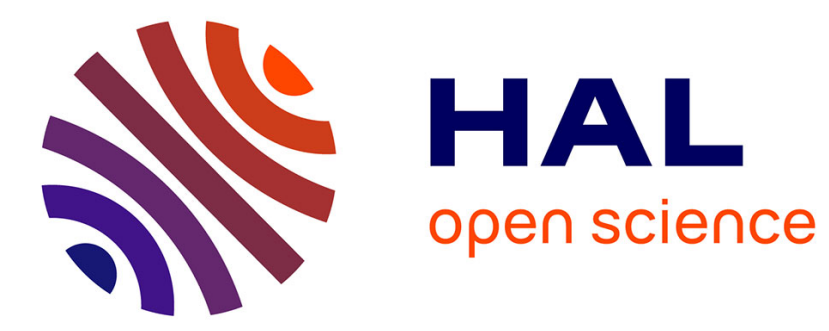

\title{
X-ray lensless microscopy from undersampled diffraction intensities
}

\author{
F. Berenguer, Pierre Godard, Marc Allain, J.-M. Belloir, A. Talneau, S. Ravy, \\ Virginie Chamard
}

\section{- To cite this version:}

F. Berenguer, Pierre Godard, Marc Allain, J.-M. Belloir, A. Talneau, et al.. X-ray lensless microscopy from undersampled diffraction intensities. Physical Review B: Condensed Matter and Materials Physics (1998-2015), 2013, 88, pp.144101-1. 10.1103/PhysRevB.88.144101 . hal-00870328

\section{HAL Id: hal-00870328 \\ https://hal.science/hal-00870328}

Submitted on 26 Sep 2015

HAL is a multi-disciplinary open access archive for the deposit and dissemination of scientific research documents, whether they are published or not. The documents may come from teaching and research institutions in France or abroad, or from public or private research centers.
L'archive ouverte pluridisciplinaire HAL, est destinée au dépôt et à la diffusion de documents scientifiques de niveau recherche, publiés ou non, émanant des établissements d'enseignement et de recherche français ou étrangers, des laboratoires publics ou privés. 


\title{
X-ray lensless microscopy from undersampled diffraction intensities
}

\author{
F. Berenguer, ${ }^{1}$ P. Godard, ${ }^{2,}{ }^{*}$ M. Allain, ${ }^{2}$ J.-M. Belloir, ${ }^{1}$ A. Talneau, ${ }^{3}$ S. Ravy, ${ }^{1}$ and V. Chamard ${ }^{2}$ \\ ${ }^{1}$ Synchrotron SOLEIL, L'Orme des merisiers, Saint-Aubin BP 48, 91192 Gif-sur-Yvette cedex, France \\ ${ }^{2}$ Aix Marseille Université, CNRS, Centrale Marseille, Institut Fresnel, UMR 7249, 13013 Marseille, France \\ ${ }^{3}$ CNRS-Laboratoire de Photonique et de Nanostructures, F-91460 Marcoussis, France
}

(Received 7 June 2013; revised manuscript received 19 July 2013; published 1 October 2013)

\begin{abstract}
$\mathrm{X}$-ray coherent diffraction imaging including ptychography provides the nanoscale resolved three-dimensional description of matter. The combination of these approaches to the Bragg geometry case arouses a strong interest for its capability to provide information about strain state in crystals. Among the existing approaches, ptychography is particularly appealing because it allows the investigation of extended or weakly scattering samples. Coherent diffraction imaging approaches, based on redundancy in the collected diffraction intensity data set, are highly time consuming and rely on state-of-the-art mechanical setups, both being strong limitations for a general application. We show here that these can be overcome by regularization-based inversion algorithms introducing a priori structural knowledge. This method, which can be generalized to other wavelengths or beam sources, opens new possibilities for the imaging of radiation-sensitive specimens or very large samples.
\end{abstract}

DOI: 10.1103/PhysRevB.88.144101

PACS number(s): 61.05.cp, 42.30.Rx, 61.46.-w, 68.37.Yz

\section{INTRODUCTION}

Properties of nanomaterials are strongly entangled to their structural behavior at the nanoscale. This is particularly relevant for crystals where the strain state can be used to design specific functionalities like the modification of the energy bands in semiconductors. ${ }^{1}$ Hence, progress in nanoscience calls for the development of imaging methods at this scale. However, mapping the strain fields inside a threedimensional (3D) crystal remains challenging. Transmission electron microscopy offers atomic resolution, but requires the thinning down of the sample. ${ }^{2}$ On the other hand, $x$ rays allow in operando characterization of strain fields: Diffraction intensity data are collected in the vicinity of a Bragg reflection and further converted into an image. Scanning diffraction with a nanofocused beam provides two-dimensional (2D) information whose interpretation is based on a structural model. ${ }^{3}$ On the contrary, coherent diffraction imaging (CDI) aims at retrieving the quantitative $3 \mathrm{D}$ information from a set of diffracted intensities by phasing back the diffracted field with inversion algorithms and propagating it up to the image space. ${ }^{4,5}$ When performed in the vicinity of a Bragg reflection, the retrieved direct space quantity is a complex-valued function, whose phase provides information about the crystalline displacement field. For a sample known to be isolated and smaller than the beam coherence length, the phase problem is solved through an oversampling of the diffraction pattern. ${ }^{6}$ Ptychography overcomes the limits encountered in finite-support phase-retrieval approaches. It consists of scanning a sample across a finitesize illumination and measuring partially redundant coherent diffraction intensity patterns corresponding to overlapping illuminated areas. ${ }^{7}$ Three-dimensional Bragg ptychography has already been presented in Refs. 8 and 9. One-dimensional strain fields and 2D polarization maps obtained with 2D Bragg ptychography are favorably compared with numerical simulations in Refs. 10 and 11, respectively.

The major difficulty encountered in these approaches results from the need to acquire a large data set. This is particularly critical for $3 \mathrm{D}$ (Bragg) ptychography, which requires $3 \mathrm{D}$ measurements for each sample position. ${ }^{8,12}$ This implies long acquisition time, on the order of several hours. As a consequence, the application of the technique is limited by radiation damage and sample positional drift. Furthermore, the sampling that is necessary in the third direction imposes the value of the rocking curve angular step on the order of a millidegree, reaching sometimes the mechanical limits of the diffractometer. A solution to overcome these problems is the Bragg projection ptychography, where a $2 \mathrm{D}$ projection of the sample image is reconstructed by collecting 2D (only) diffraction patterns. ${ }^{10,11,13}$ However, for samples requiring 3D imaging, the encountered experimental limits result in a detrimental sparsity in the Fourier space. Therefore, solving the 3D case necessarily goes with the retrieval of that missing Fourier information. It can be done through the introduction of a priori information concerning the object to retrieve. According to Bayesian statistic inference literature, this is equivalent to regularization. Hence, it is not a surprise that regularization techniques are able to partially compensate for missing information in the data set. Very similar effects are denoted, for example, in irregular sampling ${ }^{14}$ or in medical magnetic resonance image reconstruction. ${ }^{15}$

Here, we show that regularization techniques can be combined to $3 \mathrm{D}$ CDI in order to provide a 3D image of the sample, in spite of missing Fourier information. We demonstrate the relevance of this approach on numerical and experimental data, for which the oversampling ratio is limited to 0.25 in one direction.

\section{REGULARIZATION AND 3D BRAGG COHERENT DIFFRACTION IMAGING}

In the ptychography approach, the sample scattering function $\rho$ and the illumination function, denoted by $P_{j}$ at the $j$ th sample-to-beam position, have to be introduced. These quantities result in the description of the exit field $\psi_{j}$ just after the sample. $\psi_{j}$ is given by the multiplicative approximation $\psi_{j}=P_{j} \rho$ as long as the interaction of $\mathrm{x}$ rays with the 
scattering volume is weak. For a noise-free hypothetical measurement, the diffraction pattern at the $j$ th position is $I_{j}=\left|\Psi_{j}\right|^{2}=\left|\mathcal{F} \psi_{j}\right|^{2}$, where $\mathcal{F}$ is the propagation operator between the sample space and the detector space. When the far-field approximation applies, this operator reduces to a simple Fourier transform.

A complete ptychographical data set consists in the diffraction patterns $\left\{I_{j}\right\}$ obtained for all sample positions $j \in\{1, \ldots, J\}$. Several methods are at hand for inverting such a set. ${ }^{7,16,17}$ Here, the scaled gradient algorithm introduced in Ref. 18 is used to minimize the cost function,

$$
\mathcal{L}(\rho)=\sum_{j}\left\|I_{j}^{1 / 2}-\left|\mathcal{F} \psi_{j}(\rho)\right|\right\|^{2},
$$

with respect to $\rho$. In order to ensure global convergence to a local minimizer, the step length between two iterations is adapted with a backtracking line search. ${ }^{19}$

In practice, the minimization of $\mathcal{L}(\rho)$ is likely to lead to multiple solutions that can be explored with the use of different starting estimates. In order to decrease the number of equivalent solutions, it becomes natural to introduce some $a$ priori knowledge, forcing the solution to present a specific (physical) property $\mathcal{A}$. This is the aim of regularization techniques, among which the most direct method consists of projecting after each minimization of $\mathcal{L}$ the updated solution on the set of all functions fulfilling $\mathcal{A}$. However, this method generally stagnates without succeeding in finding an acceptable solution. ${ }^{20}$ In order to enlarge the search space, one can make it possible to accept solutions that do not strictly satisfy $\mathcal{A}$. This is achieved by adding a term $\mathcal{R}(\rho)$ containing the a priori information to the cost function [Eq. (1) ]. ${ }^{21,22}$ The expression to minimize (with respect to $\rho$ ) then reads

$$
\mathcal{J}_{\mu}(\rho)=\mathcal{L}(\rho)+\mu \mathcal{R}(\rho),
$$

where the regularization term $\mathcal{R}(\rho)$ is minimal if $\rho$ satisfies $\mathcal{A}$. In Eq. (2), the parameter $\mu$ controls the trade-off in the solution between the data-related and the a priori information. In practice, it is standard that $\mu$ is adjusted so that the contributions of $\mathcal{L}$ and $\mathcal{R}$ have the same magnitude. ${ }^{23,24}$ In the following, we show that this regularization approach can be used to compensate for missing information in the data set. This demonstration is performed in the framework of Bragg CDI.

In Bragg CDI, the 3D image of a crystal is an effective electron density $\rho=|\rho| \exp (i \phi)$, where $|\rho|$ is the density distribution of the crystalline part contributing to the intensity at a given Bragg vector $\mathbf{G}_{h k l}$, with indexes $(h k l)$. The displacement field $\mathbf{u}$ is introduced through the phase as $\phi=\mathbf{u} \cdot \mathbf{G}_{h k l}$ (Ref. 25). Figure 1 presents the Bragg CDI measurement geometry, where for simplicity a symmetric reflection is probed. The intensity is measured as a function of the wave-vector transfer $\mathbf{q}=\mathbf{k}_{f}-\mathbf{k}_{i}$, where $\mathbf{k}_{i, f}$ are the incident and exit wave vectors, respectively. The 3D intensity acquisition results from the stacking of $2 \mathrm{D}$ patterns collected along the rocking curve. ${ }^{26}$ Because the whole range of incident angles usually fits in less than a degree, the 2D slices are assumed to be parallel and equally spaced. This sustains a detection frame $\left(\mathbf{q}_{1}, \mathbf{q}_{2}, \mathbf{q}_{3}\right)$ with $\mathbf{q}_{1}$ and $\mathbf{q}_{2}$ in the detector plane $\left(\mathbf{q}_{2}\right.$ being perpendicular to the incidence plane) and $\mathbf{q}_{3}$ along the rocking curve direction. For $i \in\{1,2,3\}, \mathbf{q}_{i}$ is a unitary vector and the sampling in this direction is denoted by $\delta q_{i}$.

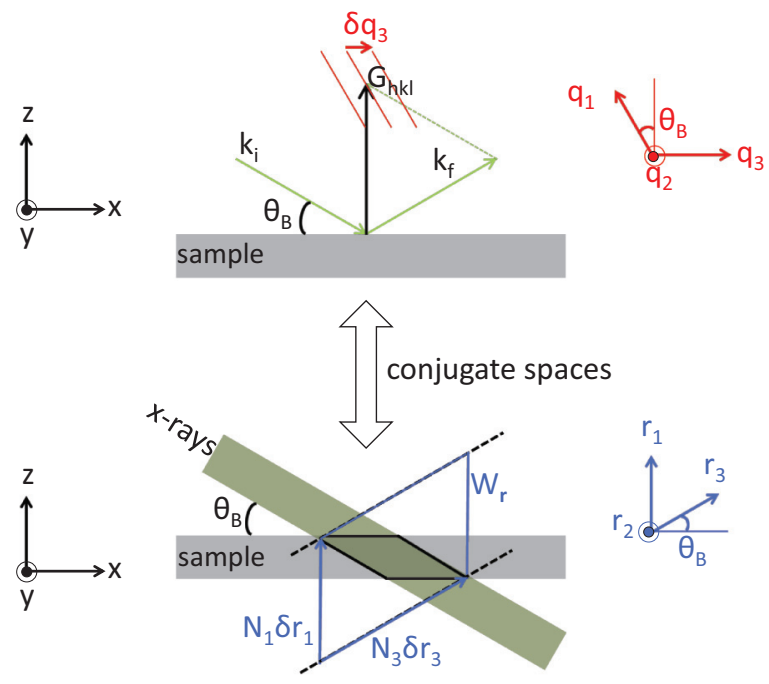

FIG. 1. (Color online) The different bases involved in Bragg CDI. The sample related basis is $(\mathbf{x}, \mathbf{y}, \mathbf{z}) ; \theta_{B}$ denotes the Bragg angle corresponding to the Bragg vector $\mathbf{G}_{h k l}$. (Top) The measurement basis is $\left(\mathbf{q}_{1}, \mathbf{q}_{2}, \mathbf{q}_{3}\right)$, with the first two directions along the area detector and the last one being probed as the sample is tilted along the rocking curve. (Bottom) The conjugate of this basis is $\left(\mathbf{r}_{1}, \mathbf{r}_{2}, \mathbf{r}_{3}\right)$. The blue frame $W_{\mathbf{r}}$ is the smallest numerical window which still fully contains the exit field, represented in dark green.

$N_{i}$ is the number of pixels in the corresponding direction. The volume of the whole measurement window $W_{\mathbf{q}}$ is then $\left|W_{\mathbf{q}}\right|=\left[\left(N_{1} \delta q_{1} \mathbf{q}_{1}\right) \times\left(N_{2} \delta q_{2} \mathbf{q}_{2}\right)\right] \cdot\left(N_{3} \delta q_{3} \mathbf{q}_{3}\right)$.

In a phase-retrieval approach, the field is propagated back and forth between the detection space and the sample space, using a Fourier-transform-based propagator. Practically, the use of a discrete Fourier transform requires, in the sample space, the introduction of $W_{\mathbf{r}}$, the conjugate of $W_{\mathbf{q}}$. The associated direct space frame, $\left(\mathbf{r}_{1}, \mathbf{r}_{2}, \mathbf{r}_{3}\right)$, is obtained by the conjugation relations $\mathbf{r}_{i}=2 \pi\left(N_{j} \delta q_{j} \mathbf{q}_{j}\right) \times\left(N_{k} \delta q_{k} \mathbf{q}_{k}\right) /\left|W_{\mathbf{q}}\right|$, where $(i, j, k)$ is one of the three circular permutations of $(1,2,3)$. The sampling in $W_{\mathbf{r}}$ is given by

$$
\delta r_{i}=\left|\mathbf{r}_{i}\right|=\frac{2 \pi\left|\mathbf{q}_{j} \times \mathbf{q}_{k}\right|}{N_{i} \delta q_{i}\left[\left(\mathbf{q}_{j} \times \mathbf{q}_{k}\right) \cdot \mathbf{q}_{i}\right]} .
$$

In particular, the size of $W_{\mathbf{r}}$ along $\mathbf{r}_{3}$ is given by $N_{3} \delta r_{3}=$ $2 \pi /\left[\delta q_{3} \cos \left(\theta_{B}\right)\right]$, where $\theta_{B}$ denotes the Bragg angle. In order to avoid aliasing in the reconstructed image, the exit field $\psi_{j}$ has to be fully contained into $W_{\mathbf{r}}$, as shown in Fig. 1 . It results in a strong constraint onto $\delta q_{3}$. In the following, we show that our regularization technique is able to compensate for a highly sparse sampling along the $\mathbf{q}_{3}$ direction.

\section{NUMERICAL DEMONSTRATION}

Figures 2(a) and 2(b) present the sample designed for the numerical demonstration of the regularization test. We chose a set of two lines, one being infinite and the other one truncated. Their edges are slightly inclined. The thickness and width of the lines are 0.18 and $0.95 \mu \mathrm{m}$, respectively and the edge-to-edge distance is $2 \mu \mathrm{m}$. Furthermore, as the lines are strain free, their respective phases are set to zero. The probe, shown in Fig. 4(a), corresponds to the one used 


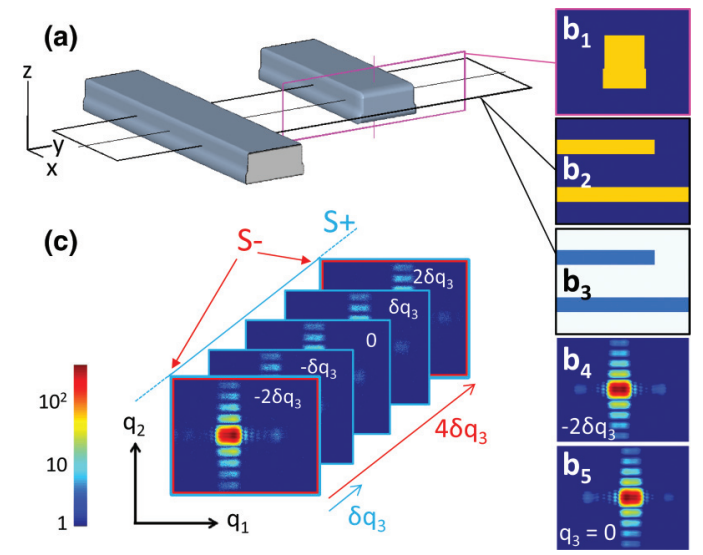

FIG. 2. (Color online) Numerical sample. (a) Three-dimensional view of the numerical object. The axis black lines are $0.4 \mu \mathrm{m}$ long. $\left(b_{1}\right),\left(b_{2}\right)$ Modulus of the test object shown in the $(y, z)$ and $(x, y)$ planes, indicated in color in (a) (in arbitrary units). ( $\left.b_{3}\right)$ Phase (in radians) shown in the $(x, y)$ plane. $\left(b_{4}\right),\left(b_{5}\right)$ Diffraction patterns in $\left(\mathbf{q}_{1}, \mathbf{q}_{2}\right)$ at $\mathbf{q}_{3}=-2 \delta q_{3}$ and $\mathbf{q}_{3}=0$, respectively. (c) Photon shot noise corrupted diffraction intensity patterns taken along the rocking curve with the beam centered between the lines (in photons). $\mathcal{S}_{+}$is the full data set while $\mathcal{S}_{-}$extracts one over four diffraction patterns from $\mathcal{S}_{+}$. The arrow length represents $50 \mu \mathrm{m}^{-1}$.

in the experiment described further. The field has a typical central spot size of about $3 \times 1 \mu \mathrm{m}^{2}$ (horizontal $\times$ vertical). The scanning of the sample with regard to the beam position corresponds as well to the experimental conditions: It consists of 11 steps along the $\mathbf{r}_{2}$ direction, irregularly spaced on a total range of $4.3 \mu \mathrm{m}$. Two sets of data are defined, namely $\mathcal{S}_{+}$and $\mathcal{S}_{-}$[Fig. 2(c)]. $\mathcal{S}_{+}$corresponds to an oversampling ratio along $\mathbf{q}_{3}$ equal to 1 . Hence, $W_{\mathbf{r}}$ contains fully but tightly the exit field. On the contrary, $\mathcal{S}_{-}$corresponds to a strong decrease, by a factor of 4 , of the sampling along $\mathbf{q}_{3}$, resulting in an undersampled data set. Practically, $\mathcal{S}_{-}$is obtained by extracting only one of four diffraction planes from the $S_{+}$data set. Finally, all generated diffraction patterns are corrupted with Poisson shot noise, where a maximum of 800 photons per pixel is considered, according to the experimental observations. The whole set of data, either $\mathcal{S}_{+}$or $\mathcal{S}_{-}$, is used for the inversion in order to reconstruct the sample electron density. The initial sample guess is designed with a correct thickness but an underestimated width $(0.75 \mu \mathrm{m})$ and no truncation. Each result presented herein was obtained after 500 iterations.

The result obtained with $\mathcal{S}_{+}$is shown in Fig. 3(a). As expected, the truncation and the correct width are reconstructed. The retrieved intensity patterns are in good agreement with the measurements. This reconstruction stands as our reference reconstruction. Figure 3(b) presents the result obtained with the undersampled data set $\mathcal{S}_{-}$. To avoid aliasing, the three over four unmeasured patterns are let free to float. The retrieved solution is of poor quality: The truncation is not retrieved and the linewidth remains equal to the initial one. One observes a high discontinuity between the known and the interpolated diffraction patterns. In particular, one can note that the interference fringes retrieved in the nonmeasured plane are too large. This test shows that the method proposed in Ref. 27 cannot compensate for the missing data along the rocking

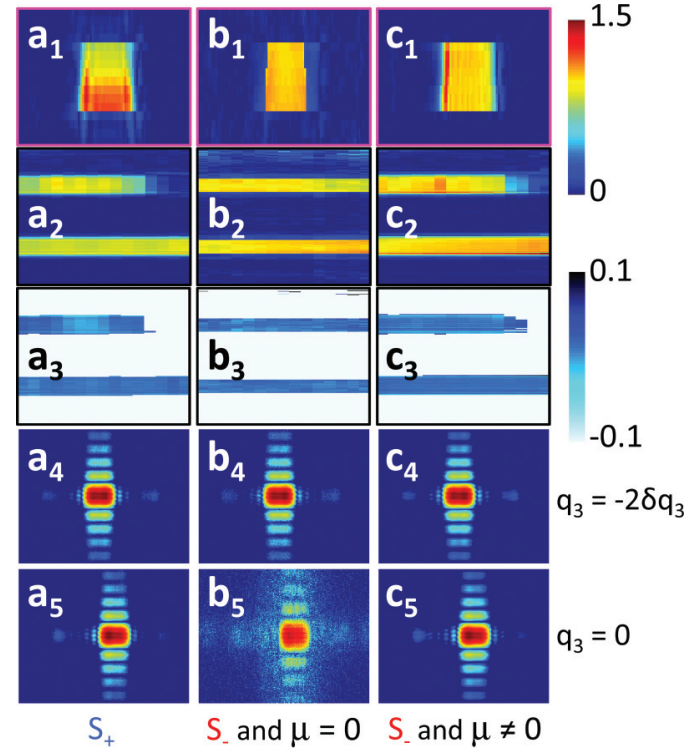

FIG. 3. (Color online) Regularization on numerical data. (a) Same as Fig. 2(b) for the reconstructed object obtained from the $\mathcal{S}_{+}$data set. (b) Same as (a) for the reconstructed object obtained from the undersampled data set $\mathcal{S}_{-}$. (c) Same as (b) introducing the regularization based on the support of the sample.

curve. The present case is arguably more demanding: The signal-over-noise ratio is rather low and, more importantly, the beam is not scanned along the undersampled direction.

The regularization term is now introduced, based on the a priori knowledge of the sample thickness. The proposed constraint acts as a soft penalization on the reconstructed density inside $T$, where $T$ is the support complementary to the planar film containing the sample. Hence, the chosen regularization term is

$$
\mathcal{R}(\rho)=\sum_{n \in T}\left|\rho_{n}\right|^{2},
$$

where $n$ is the pixel index in $W_{\mathbf{r}}$. This condition is particularly efficient in the Bragg CDI geometry: As seen in Fig. 1, $\mathbf{r}_{1}$ and $\mathbf{r}_{3}$ are not orthogonal, and thus using a constraint along the sample thickness provides a constraint along $\mathbf{r}_{3}$ as well. The result obtained with $\mathcal{S}_{-}$together with the regularization term $\left(\mu=10^{4}\right)$ leads to the reconstruction shown in Fig. 3(c). The retrieved density is in good agreement with our reference solution obtained with $\mathcal{S}_{+}$[Fig. 3(a)]. In addition, the regularization clearly improves the width of the sample in the $\mathbf{y}$ direction though the a priori information is fully independent of that direction. The retrieved phase field, which holds the displacement field information, if any, is almost constant as expected. Small fluctuations on the order of \pm 0.03 radian are observed. This value expresses the sensitivity of the proposed approach with regard to the displacement field reconstruction capability. In the measured as well as in the unmeasured planes, the retrieved intensity patterns are in good agreement with the original measurements. This last result shows that the regularization techniques are able to compensate for missing information in case of undersampled data. 

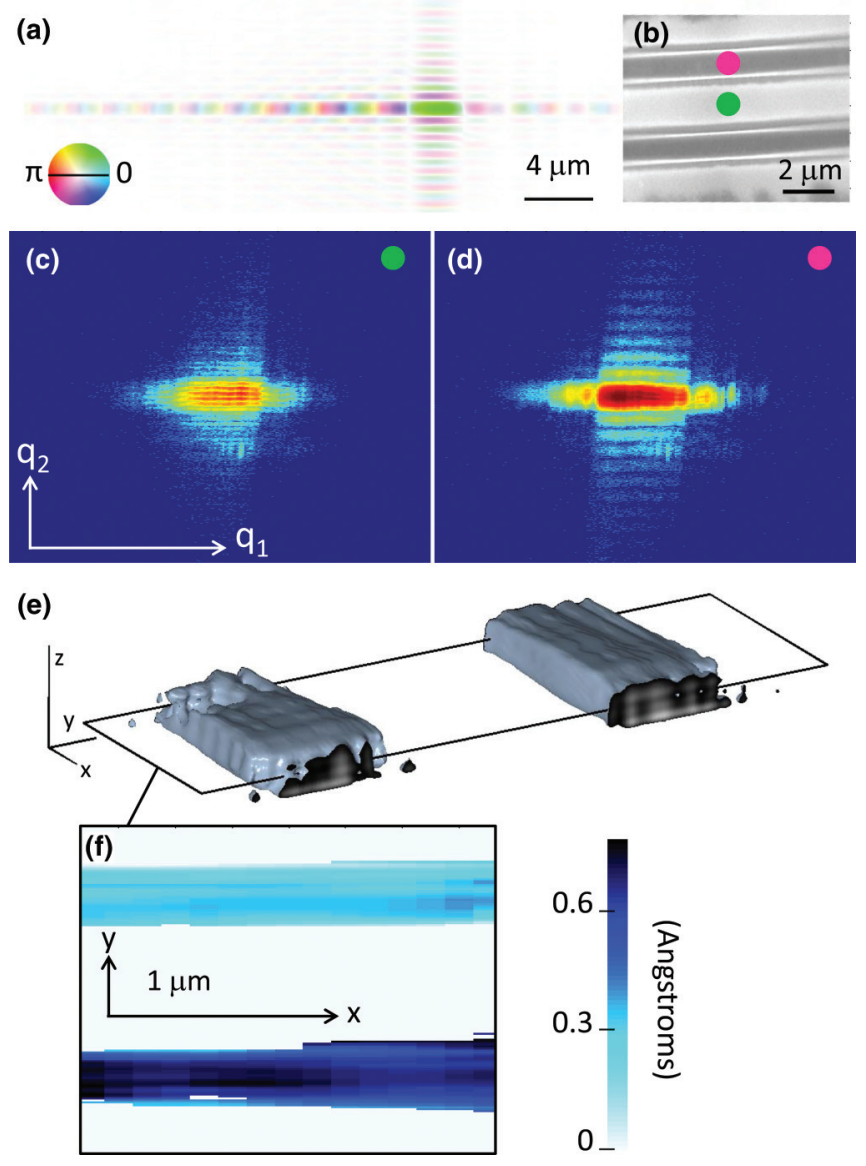

FIG. 4. (Color online) Regularization on undersampled experimental data. (a) The illumination function $P$ in the focal plane. The brightness represents $|P|$ on a logarithmic scale, whereas the color represents its phase. (b) A scanning electron microscopy top view of the two Si lines. The green and pink dots represent two beam positions. (c) and (d) Measured diffraction pattern at the center of the rocking curve, with beam at the green-dot and pink-dot positions, respectively. The arrow length is $30 \mu \mathrm{m}^{-1}$. (e) Three-dimensional isosurface of $|\rho|$ retrieved from the under-sampled data set using regularization. The axis black lines are $0.4 \mu \mathrm{m}$ long. (f) Two-dimensional cross section showing the retrieved displacement field component.

\section{EXPERIMENTAL DEMONSTRATION}

This approach is further tested onto experimental data, obtained at CRISTAL beamline (Soleil). A monochromatic beam, with an energy of $8.5 \mathrm{keV}$, is focused at the sample position using a Fresnel zone plate (FZP) with a focal length of $0.2 \mathrm{~m}$. In order to ensure beam full coherence, a set of slits, positioned off axis and located $0.135 \mathrm{~m}$ upstream of the FZP, is used. Its aperture matches the coherence length $\left[20 \times 60 \mu \mathrm{m}^{2}\right.$, along the horizontal $(\mathrm{H})$ and vertical $(\mathrm{V})$ directions, respectively]. Prior to the ptychography experiment, the illumination function complex-valued wave field is retrieved using the Fresnel-CDI methods described in Refs. 28 and 29 [Fig. 4(a)]. Data for this reconstruction are obtained by measuring the probe diffraction patterns with a $1.45-\mu \mathrm{m}$-pixel SOLEIL custom-made camera ${ }^{30}$ located $2 \mathrm{~m}$ downstream the FZP focus position. This small pixel size is needed in order to finely sample the Fresnel diffraction pattern produced by the scattering from the entrance slit.
The sample is positioned in Bragg condition onto a diffractometer, while its accurate positioning with regard to the beam spot is performed with a piezo stage (Physik Instrumente, PI). The sample diffraction patterns are recorded with a second detector with bigger active area, an Andor charge-coupleddevice detector located $2 \mathrm{~m}$ from the sample. The $1024 \times 1024$ pixels have a size of $13 \times 13 \mu \mathrm{m}^{2}$. The sample consists of a lithographically fabricated Si-on-insulator set of two parallel lines [Fig. 4(b)]. The Si(110) upper layer, whose thickness is $0.18 \mu \mathrm{m}$, is of different orientation from the $\mathrm{Si}(001)$ substrate. The width of the lines and the edge-to-edge distance are 1 and $2 \mu \mathrm{m}$, respectively. On average, the patterned lines are expected to be strain free. A ptychographical data set is obtained, in the vicinity of the 220 upper layer Bragg reflection $\left(\theta_{\mathrm{B}}=22.32^{\circ}\right)$, by scanning the sample perpendicularly across the lines. A total of 11 positions is used with a step size of about $0.5 \mu \mathrm{m}$. The exposure time is $50 \mathrm{~s}$ for each diffraction pattern. The chosen angular step of $0.01^{\circ}$ together with the 8 steps along the rocking curve result in a total angular range of $0.09^{\circ}$. A cross section of two different 3D diffraction patterns is presented in Figs. 4(c) and 4(d). These experimental conditions correspond to an undersampled data set. Precisely, the sampling ratio along the $\mathbf{q}_{3}$ is only 0.25 .

The whole $4 \mathrm{D}$ data set is inverted using the function $\mathcal{J}_{\mu}$ with the regularization term given by Eq. (4) and $\mu=10^{3}$. The reconstructed object is presented in Figs. 4(e) and 4(f). The two lines are clearly identified, with width and separation in good accordance with the SEM image. We note, however, that the line at large $y$ has a better defined shape. This likely results from the values chosen for determining the scan center and extent during the ptychography acquisition. Indeed, this scan was centered onto the line located at large $y$. Hence, the reconstruction is spatially of different quality, according to the amount of information collected for each sample area. The displacement field image shows that the two lines are vertically shifted by about $0.4 \AA$, which could result from the presence of a waviness at the interface between the upper layer and the substrate as observed in Ref. 31. The resolution along $\mathbf{y}$ is roughly estimated on the order of $0.08 \mu \mathrm{m}$. This value is in good agreement with the resolution obtained during the numerical study presented in Fig. 3( $\left.a_{1}\right)$, using the full data set. One can note that the resolution should improve if a strongly scattering material (like a metallic $\mathrm{crystal}^{5}$ ) is investigated, as long as the mechanical instabilities of the setup remain negligible. Along the other directions, the experimental estimation of the resolution is not relevant: The object does not present any edge or fine structure along the $x$ axis, which would be needed to quantify the resolution. Along the $z$ axis, the resolution measured at the surface edge depends on the $a$ priori information and on the regularization.

\section{DISCUSSION AND CONCLUSIONS}

The use of a ptychography approach is not only appealing for tackling the case of extended samples. It allows in addition the use of a highly focused beam, with an extent smaller than the sample. It increases thereby the coherent flux at the sample position and, consequently, the signal-to-noise ratio in the diffraction pattern. Considering the weak scattering power of silicon, one can foresee that the 3D imaging of 
a silicon crystalline structure like the one presented here is hard to obtain with plane-wave illumination and support-based CDI approaches. However, the development of ptychography suffers from encountered mechanical difficulties and extensive acquisition time. Here we have shown that the regularization techniques within Bragg ptychography are able to compensate for an undersampled data set.

The knowledge of the support along the direction $\mathbf{r}_{1}$ is particularly well suited in the present case. This favorable situation results from the combination of the two following points. First, the direction $\mathbf{q}_{1}$ is very well sampled. Hence, introducing the regularization results in a constraint which applies onto a large number of pixels in the direct space. In addition, the direction $\mathbf{r}_{1}$ cannot be scanned during the ptychography acquisition, due to a lack of diversity of the probe along the beam propagation direction. Therefore, the support constraint, which holds onto the direction $\mathbf{r}_{1}$, brings the needed information about the sample position, which is indeed not contained into the data set. It prevents the presence of echoes which are likely to appear along the $\mathbf{r}_{1}$ direction. Moreover, the Bragg angle is sufficiently large for this constraint to have a pronounced effect on the information recovery along $\mathbf{q}_{3}$. For thicker objects or smaller Bragg angles, other constraints are to be applied. Indeed, any kind of a priori information can be used: a low-resolution sample image, a specific physical property. The regularization approach can thus be generalized to other phase-retrieval-based lensless microscopy methods like transmission ptychography or support-based algorithms. In order to draw the limits of the method and investigate, for instance, the use of loose support, further work is needed. As well, the possibility to apply this approach to the investigation of strain field in crystals deserves specific extended investigation. However, the perspectives opened by the present work are of wide impact because it makes it possible to overcome several problems encountered usually in CDI. One can mention the possibility to adapt the geometry of the illumination function with regard to an eventual anisotropic sample shape: This improvement was so far limited by the symmetric sampling imposed in the detector area by the square shape of the camera pixels. Moreover, it makes it possible to get rid of the mechanical limits imposed by the instrument, when extended exit fields are considered. Finally, the strong reduction of the total illumination time opens the way to the imaging of radiation-sensitive samples like biological specimens.

For all these reasons, we believe that regularization techniques will constitute a clear breakthrough in the development of 3D nanoscale imaging.

\section{ACKNOWLEDGMENTS}

We acknowledge L. Capello for providing the sample substrate. This work is funded by the French ANR under Project No. ANR-11-BS10-0005. *pierre.godard@fresnel.fr

${ }^{1}$ M. J. Süess, R. Geiger, R. A. Minamisawa, G. Schiefer, J. Frigerio,

D. Chrastina, G. Isella, R. Spolenak, J. Faist, and H. Sigg, Nat.

Photon. 7, 466 (2013).

${ }^{2}$ M. Hÿtch, F. Houdellier, F. Hüe, and E. Snoeck, Nature (London) 453, 1086 (2008).

${ }^{3}$ C. Mocuta, J. Stangl, K. Mundboth, T. H. Metzger, G. Bauer, I. A. Vartanyants, M. Schmidbauer, and T. Boeck, Phys. Rev. B 77, 245425 (2008).

${ }^{4}$ J. Miao, T. Ishikawa, B. Johnson, E. H. Anderson, B. Lai, and K. O. Hodgson, Phys. Rev. Lett. 89, 088303 (2002).

${ }^{5}$ M. A. Pfeifer, G. J. Williams, I. A. Vartanyants, R. Harder, and

I. K. Robinson, Nature (London) 442, 63 (2006).

${ }^{6}$ D. Sayre, Acta Crystallogr. 5, 843 (1952).

${ }^{7}$ J. M. Rodenburg and H. M. L. Faulkner, Appl. Phys. Lett. 85, 4795 (2004).

${ }^{8}$ P. Godard, G. Carbone, M. Allain, F. Mastropietro, G. Chen, L. Capello, A. Diaz, T. H. Metzger, J. Stangl, and V. Chamard, Nat. Commun. 2, 568 (2011).

${ }^{9}$ X. Huang, R. Harder, S. Leake, J. Clark, and I. K. Robinson, J. Appl. Crystallogr. 45, 778 (2012).

${ }^{10}$ S. O. Hruszkewycz, M. V. Holt, C. E. Murray, J. Bruley, J. Holt, A. Tripathi, O. G. Shpyrko, I. McNulty, M. J. Highland, and P. H. Fuoss, Nano Lett. 12, 5148 (2012).

${ }^{11}$ S. O. Hruszkewycz, M. J. Highland, M. V. Holt, D. Kim, C. M. Folkman, C. Thompson, A. Tripathi, G. B. Stephenson, S. Hong, and P. H. Fuoss, Phys. Rev. Lett. 110, 177601 (2013).

${ }^{12}$ M. Dierolf, A. Menzel, P. Thibault, P. Schneider, C. M. Kewish, R. Wepf, O. Bunk, and F. Pfeiffer, Nature (London) 467, 436 (2010).
${ }^{13}$ Y. Takahashi, A. Suzuki, S. Furutaku, K. Yamauchi, Y. Kohmura, and T. Ishikawa, Phys. Rev. B 87, 121201 (2013).

${ }^{14}$ S. Bourguignon, H. Carfantan, and T. Böhm, Astron. Astrophys. 462, 379 (2007).

${ }^{15}$ R. Boubertakh, J. F. Giovannelli, A. De Cesare, and A. Herment, Signal Process. 86, 2479 (2006).

${ }^{16}$ M. Guizar-Sicairos and J. R. Fienup, Opt. Express 16, 7264 (2008).

${ }^{17}$ P. Thibault, M. Dierolf, O. Bunk, A. Menzel, and F. Pfeiffer, Ultramicroscopy 109, 338 (2009).

${ }^{18}$ P. Godard, M. Allain, V. Chamard, and J. M. Rodenburg, Opt. Express 20, 25914 (2012)

${ }^{19}$ J. Nocedal and S. J. Wright, Numerical Optimization, 2nd ed. (Springer, Berlin, 2006), p. 41.

${ }^{20} \mathrm{This}$ is reminiscent of the error-reduction algorithm that most probably falls into a local minimum that is quite far from the global minimum.

${ }^{21} \mathrm{G}$. Demoment, IEEE Trans. Acoust., Speech Signal Process. ASSP 37, 2024 (1989).

${ }^{22}$ P. Godard, M. Allain, and V. Chamard, Phys. Rev. B 84, 144109 (2011).

${ }^{23}$ P. C. Hansen, SIAM Rev. 34, 561 (1992).

${ }^{24}$ M. Allain and J. P. Roques, Astron. Astrophys. 447, 1175 (2006).

${ }^{25}$ I. K. Robinson and R. Harder, Nat. Mater. 8, 291 (2009).

${ }^{26}$ G. J. Williams, M. A. Pfeifer, I. A. Vartanyants, and I. K. Robinson, Phys. Rev. Lett. 90, 175501 (2003).

${ }^{27}$ T. B. Edo, D. J. Batey, A. M. Maiden, C. Rau, U. Wagner, Z. D. Pesic, T. A. Waigh, and J. M. Rodenburg, Phys. Rev. A 87, 053850 (2013). 
${ }^{28}$ H. Quiney, A. Peele, Z. Cai, D. Paterson, and K. Nugent, Nat. Phys. 2, 101 (2006).

${ }^{29}$ F. Mastropietro, D. Carbone, A. Diaz, J. Eymery, A. Sentenac, T. H. Metzger, V. Chamard, and V. Favre-Nicolin, Opt. Express 19, 19223 (2011).
${ }^{30}$ M. Bourdessoule, J. Phys.: Conf. Ser. 425, 192018 (2013).

${ }^{31}$ A. A. Minkevich, M. Gailhanou, J. S. Micha, B. Charlet, V. Chamard, and O. Thomas, Phys. Rev. B 76, 104106 (2007). 